\title{
Tomada de Decisão com Base em Moving Average Convergence-Divergence: Uma Análise sobre Volatilidade
}

\section{Decision Making Based on Moving Average Convergence-Divergence: A Volatility Analysis}

\author{
Valdinei Simas Egresso do MBA em Administração Financeira e Controladoria. Universidade do Vale do Itajaí \\ https://orcid.org/0000-0001-5095-8696 (Univali) - Brasil.valdinei.simas@uol.com.br \\ Raul Beal Partyka Doutorando na Escola de Administração de Empresas da Fundação Getulio Vargas (FGV EAESP) \\ https://orcid.org/0000-0001-7941-2152 - Brasil. raul.partyka@fgv.edu.br \\ Jeferson Lana Doutor em Administração de Empresas pela Fundação Getulio Vargas (FGV EAESP) - Brasil. \\ https://orcid.org/0000-0002-9787-1114 jlana@univali.br
}

\section{RESUMO}

Este estudo contempla o movimento crescente de investidores saindo da renda fixa e iniciando no mercado de renda variável, muitos dos quais utilizam técnicas simples como o Moving Average ConvergenceDivergence(MACD) e por vezes escolhem ativos com pouca volatilidade, pois empiricamente entende que são mais "seguros". O objetivo do estudo é estimar a relação entre a intensidade da volatilidade de um ativo e a eficiência do instrumento MACD. Para atingir o objetivo, foi realizada análise descritiva - com coleta documental, e análise quantitativa - por meio da Análise de Variância (ANOVA). Buscou-se empresas que compõem determinado índice da bolsa de valores, dividindo-as em dois grupos: as $20 \%$ mais voláteis e as $20 \%$ menos voláteis. Foram realizadas simulações de compras e vendas para identificar os retornos obtidos. É possível identificar que o grupo dos ativos mais voláteis obteve resultados - lucro - exponencialmente melhores do que o outro, realizando assim a contribuição teórica e empírica das regras técnicas de negociação, especialmente via análise gráfica e utilização do instrumento MACD, sobretudo, comprovando que a relação tem capacidade preditiva no Brasil. Os resultados do estudo apoiam o emprego das técnicas, as quais promovem retornos excedentes de forma prática para os investidores. Evidências foram alcançadas da existência da relação entre a intensidade da volatilidade e a eficiência do MACD. O estudo implica na demonstração da estratégia de investimento de utilização da volatilidade como moderador da eficiência da análise gráfica.

Palavras-chave: MACD; volatilidade; análise técnica; tomada de decisão.

\section{ABSTRACT}

This study analyses the growing movement of investors leaving fixed income and moving to the variable income market, many of them use simple techniques such as Moving Average Convergence-Divergence (MACD) and sometimes choose assets with little volatility because, empirically, they see them as "safer". The purpose of the study is to estimate the relationship between the level of an asset's volatility and the efficiency of the MACD instrument. To achieve the objective, a descriptive analysis was performed - with a documentary collection, and quantitative analysis - through Analysis of Variance (ANOVA). We searched for companies that make up a given stock exchange index, dividing them into two groups: the $20 \%$ more volatile and the $20 \%$ less volatile. Simulations of purchases and sales were carried out to identify the returns obtained. It is possible to identify that the group of the most volatile assets obtained results - profit - exponentially better than the other, thus achieving the theoretical and empirical contribution of the technical rules of trading, especially via graphical analysis and use of the MACD instrument. Above all, it proved that the relationship has predictive capacity in Brazil. The results of the study support the use of techniques, which promote surplus returns in a practical way for investors. Evidence was found of the existence of a relationship between the intensity of volatility and the efficiency of the MACD. The study implies demonstrating the investment strategy of using volatility as a moderator of the efficiency of graphical analysis.

Keywords: MACD; volatility; technical analysis; decision making. 


\section{INTRODUÇÃO}

O mercado brasileiro de pequenos investidores culturalmente, em sua maioria, realiza suas aplicações em investimentos de renda fixa, em suma, na poupança (MARTIN; SBICCA, 2021). Porém, com a queda da taxa básica de juros (SELIC) e a entrada de novos players no mercado financeiro, como fintech's, corretoras de títulos e valores mobiliários - CTVM, e distribuidoras de títulos e valores mobiliários - DTVM, tem aguçado esses pequenos investidores a buscarem maiores retornos e investirem em outros mercados, como por exemplo, 0 mercado de renda variável, mais especificamente, ações (PARTYKA; LANA; GAMA, 2020).

As fintech's promovem avanços no setor financeiro, não só com tecnologia, mas também com novas estratégias do negócio (WONGLIMPIYARAT, 2017). Os bancos sempre estiveram expressivamente avançados em tecnologia, porém, a inovação está na maneira como entregam, divulgam e oferecem seus serviços, seja um banco ou outra empresa do mercado financeiro, uma fintech precisa ser simples e eficiente (DHAR; STEIN, 2017). Neste contexto, podemos entender fintech's como empresas que atuam promovendo inovações e criando uma percepção aos usuários do mercado financeiro (PARTYKA; LANA; GAMA, 2020).

As CTVM e DTVM são instituições integrantes do Sistema Financeiro Nacional - SFN, e tem por finalidade oportunizar a negociação de títulos e valores mobiliários, gerando liquidez para estes ativos (SILVA et al., 2016). A aquisição de ativos no mercado brasileiro de ações obrigatoriamente deve ser transacionada por uma CTVM/DTVM, sendo a função principal destas instituições intermediar a compra e venda de ativos entre os investidores e tomadores (FORTUNA, 2019).

No que tange os investimentos financeiros, conforme citam Bodie, Kane e Marcus (2018), existem basicamente dois tipos de análises para este mercado: a análise técnica, que trata de ferramentas de análises gráficas contando com diversos indicadores e métodos, sendo uma delas o Moving Average Convergence Divergence (MACD, do inglês Convergência e Divergência de Médias Móveis); e a análise fundamentalista, que busca uma análise das demonstrações contábeis da empresa, mercado, cenário econômico/político, entre outros fatores, portanto, uma análise mais complexa para a maioria dos usuários deste instrumento. Tanto a análise gráfica quanto a fundamentalista, contribuem com o investidor no processo de seleção de ativos de investimento.

Estes investidores, por vezes, não possuem experiência e tempo para realizar análises técnicas (VIDOTTO; MIGLIATO; ZAMBON, 2009), motivo pelo qual o presente estudo buscará evidenciar se há, ou não, relação entre o indicador de tendência $M A C D$, com a intensidade de volatilidade do ativo financeiro. Busca-se, assim, uma forma de aprimorar a análise deste pequeno investidor, que poderá optar em realizar a referida análise em ativos cuja intensidade de volatilidade tenha maior percentual de acerto.

Neste sentido, tem-se a seguinte questão problema: Qual a relação entre a intensidade de volatilidade de um ativo e a eficiência do instrumento MACD? Em outras palavras, esta pesquisa visa analisar se a eficiência de previsibilidade do MACD possui relação com a volatilidade do ativo estudado. Em caso afirmativo, este artigo tem potencial de contribuir com aspectos teóricos e empíricos nas dimensões de análise e seleção de ativos de investimento.

O presente artigo tem como objetivo geral estimar a relação entre a intensidade da volatilidade de um ativo e a eficiência do instrumento MACD. Para isto, foram selecionadas as ações que compõe o índice IBrX 100; traçada a intensidade de volatilidade das respectivas ações, separando em partes; identificado os pontos de compra e venda na análise MACD destes ativos em um período específico; verificado o lucro nas decisões tomadas, bem como a performance acima do índice Bovespa; e por fim, analisado se há relação entre a eficiência do MACD e a intensidade da volatilidade.

Explica-se a presente pesquisa para o uso de pequenos investidores que, empiricamente, estão buscando instrumentos de análise. Dentre as principais contribuições oferecidas por este artigo, podem-se citar: i) a demonstração de uma ferramenta de seleção de ativos para pequenos investidores, ii) a ponderação do uso de uma ferramenta técnica de seleção de ações a partir da volatilidade do ativo avaliado, o que pode levar a maior eficiência da ferramenta no longo prazo; iii) finalmente, uma contribuição metodológica aos antecedentes teóricos de análise técnica que não apresentam interação das ferramentas de análise com características individuais dos ativos. 


\section{REFERENCIAL TEÓRICO}

Neste tópico são apresentadas as características e evolução do quadro de investidores pessoas físicas na bolsa de valores brasileira. Além disso, será discorrido sobre o conceito de volatilidade, bem como em qual indicador de volatilidade a pesquisa será norteada. Por fim, será realizada uma explanação sobre MACD e relacionado estudos anteriores que embasam o presente estudo.

A base teórica possui embasamento no estudo de Wang et al. (2016), no qual foram realizados estudos sobre o instrumento MACD adaptado como técnica para análise do ativo petróleo em mercados futuros, onde uma das hipóteses foi a relação de variação da volatilidade. Foram realizadas pesquisas nas bases EBSCO e Google Scholar, em maio de 2018, para os termos "Moving Average Convergence-Divergence" AND "volatilidade" AND "ações" OR "ativos financeiros", para todos os anos disponíveis de estudos publicados, e não foram encontrados estudos semelhantes que utilizem esta relação, motivo pelo qual não foi possível correlacionar outros trabalhos.

\subsection{Características e evolução do quadro de investidores pessoas físicas}

Empiricamente, nota-se que o número de investidores pessoas físicas no Brasil em ativos da bolsa de valores brasileira vêm aumentando organicamente. Faz-se necessário fundamentar esses dados e, por isso, neste tópico serão apresentados dados extraídos da Bolsa, Brasil e Balcão (B3). A Tabela 1 classifica a quantidade de investidores no mercado de ações brasileiro.

Tabela 1 - Quantidade de investidores

\begin{tabular}{lcc}
\hline CLASSIFICAÇÃO & QUANT. & $\%$ \\
\hline Pessoas Físicas & 680.576 & $96,97 \%$ \\
Homens & 525.903 & $74,93 \%$ \\
Mulheres & 154.673 & $22,04 \%$ \\
Pessoas Jurídicas & 21.279 & $3,03 \%$ \\
\hline
\end{tabular}

TOTAL

$\mathbf{7 0 1 . 8 5 5}$

Nota. Contagem dos CPFs/CNPJs de investidores por Agente de Custódia, em maio de 2018. Fonte: B3 (2021).

Conforme ilustrado na Tabela 1, em maio de 2018 apresentou-se um número de 680.576 contas vinculadas a pessoas físicas no Brasil um número baixo em relação à população total e levando em consideração que pode haver pessoas com mais de uma conta. Nota-se que existe predominância de investidores homens em relação às mulheres. A proporção de investidores do sexo masculino, de 2002 até 2018 (maio), não baixou de $74 \%$. Por outro lado, o de investidoras do sexo feminino, nunca chegou a $26 \%$. Em uma análise de média para o mesmo período, tem-se que, de cada 100 investidores brasileiros, 77 são do sexo masculino e 23 são do sexo feminino.

Outro fator interessante a ser analisado é a concentração de seis Estados brasileiros em número de contas e volumes de operações (Tabela 2), os quais representam 81,68\% em números de contas, e 89,83\% em valores. Estes percentuais expressam o quão o mercado de ações brasileiro ainda pode evoluir, tendo muito espaço para isto.

Tabela 2 - Divisão por Estados

\begin{tabular}{lccccccc}
\multirow{2}{*}{ ESTADO } & \multicolumn{9}{c}{ CONTAS } & \multicolumn{3}{c}{ VALOR (R\$) } & \multirow{2}{*}{$\%$} \\
\cline { 2 - 7 } & HOMENS & MULHERES & TOTAL & HOMENS & MULHERES & TOTAL & \\
\hline SP & 219.849 & 69.808 & 289.657 & 64,03 & 20,80 & 84,84 & $47,70 \%$ \\
RJ & 78.632 & 26.814 & 105.446 & 25,96 & 11,94 & 37,90 & $21,31 \%$ \\
MG & 42.818 & 11.376 & 54.194 & 12,32 & 1,92 & 14,24 & $8,01 \%$ \\
RS & 33.329 & 8.904 & 42.233 & 7,96 & 2,90 & 10,86 & $6,11 \%$ \\
SC & 20.952 & 4.698 & 25.650 & 3,92 & 1,21 & 5,13 & $2,89 \%$ \\
PR & 30.792 & 7.945 & 38.737 & 5,15 & 1,64 & 6,79 & $3,82 \%$ \\
\hline
\end{tabular}

* Nota: Em bilhões de Reais.

Fonte: B3 (2021). 
Corroborando a ideia apresentada na introdução deste trabalho, a Tabela 2 apresenta os dados que demonstram a evolução do número de investidores pessoas físicas na bolsa de valores brasileira, tendo involução apenas nos anos de 2011, 2014 e 2015, nos demais anos sempre houve um crescimento. Em 2011 a bolsa teve uma queda de 18\% o que em parte pode explicar a involução; já em 2014 e 2015 houve crescente da taxa básica de juros (SELIC), saindo de 7,75\% no final de 2013 para 14,25\% em meados de 2015, o que foi um fator preponderante para a fuga de investidores da bolsa de valores.

\subsection{Volatilidade}

Volatilidade pode ser entendida como a oscilação dos preços de um ativo, sendo uma medida de risco, pois quanto mais um ativo oscila, em tese, mais risco ele possui (AUGUSTO; SILVA, 2012; MURCIA et al., 2011). No âmbito de volatilidade existe basicamente volatilidade histórica (Desvio padrão e média móvel), estatística (Exponentially Weighted Moving-Average - EWMA), heterocedasticidade condicional (Autoregressive Conditional Hetercedasticity - $A R C H$, e Generalized Autoregressive Conditional Hetercedasticity - GARCH), estocástica (Modelo de volatilidade estocástica - MVE) e implícita (Modelo Black \& Sholes) (PINHO; CAMARGOS; FIGUEIREDO, 2017). É possível notar que existem diversos tipos de forma para medir a volatilidade, algumas mais simples, até modelos matemáticos complexos. Os indicadores disponíveis na B3 (2021) atualmente são: o desvio padrão e o EWMA.

O desvio padrão é uma forma mais simples de cálculo, que se extrai apenas a raiz quadrada da variância (HAIR et al., 2009). Já o EWMA utiliza pesos diferentes para os valores mais recentes, assim como na média móvel ponderada (BETANCOURT BEJARANO; GARCÍA DÍAZ; LOZANO RIAÑO, 2013). Segundo Adewuyi (2016) o EWMA foi uma variação do desvio padrão feita por um banco de investimento americano (JP Morgan) a ser utilizado com a média móvel exponencial e assim utilizando os preços mais recentes, tendo em vista que o movimento mais recente de preços é a melhor forma de prever os movimentos futuros. O EWMA, assim como - MACD, traz um cálculo exponencial colocando maior peso para os valores mais recentes (CAVALERI; RIBEIRO; RIBEIRO, 2011), motivo pelo qual nesta pesquisa se optou por usar esta medida de volatilidade, que apesar de possuir um cálculo mais complexo, tem a disponibilidade de consulta de acesso público na B3, tornando-se assim de fácil utilização para os usuários deste material.

\subsection{Indicador MACD}

Existem diversos indicadores no campo de análise técnica, muitos se completam, outros fortalecem a sinalização de uma tendência indicada por um primeiro, e assim sucessivamente (LO; MAMAYSKY; WANG, 2000; SAFFI, 2003). O MACD é um indicador de tendência originalmente composto por uma linha e um sinal, e após quase duas décadas foi aprimorado pelo professor da Heriot-Watt University, Thomas Aspray, onde incluiu o histograma (VAIDYA, 2020).

Uma vez que a tradução literal para MACD é médias móveis convergentes-divergentes, a média móvel é o cálculo da média do preço de um ativo por um determinado período, como por exemplo, a média móvel de 12 dias será a soma do preço do ativo por este período dividido por 12 (VIDOTTO; MIGLIATO; ZAMBON, 2009). Já para o cálculo do MACD utiliza-se a média móvel exponencial. A fórmula faz com que se tenha maior peso nos preços mais recentes do período. Obviamente, deve-se tomar por base a cotação do preço padrão por dia do ativo, seja o preço de abertura ou de fechamento, mínima ou máxima. (MARTINS; RODRIGUES, 2018). No presente trabalho será adotado o preço de fechamento. Originalmente o MACD foi criado por Gerald Appel, tendo três médias móveis exponenciais, e em gráficos é apresentado por duas linhas, onde o cruzamento dessas linhas sinaliza momentos para entrar ou sair de um ativo, sendo que, quando a linha MACD cruza para baixo da linha sinal, aponta um momento de venda, e vice-versa (VIDOTTO; MIGLIATO; ZAMBON, 2009). Visualmente o MACD é composto por uma linha, um sinal e o histograma. A linha é caracterizada pela diferença entre as médias móveis de curto e de longo prazo do ativo; o sinal pode ser definido como o resultado das médias móveis do próprio MACD por um período menor que a média de curto prazo do ativo; por último tem- 
se o histograma, formado pela diferença entre a linha MACD e a linha de sinal (CHAVES, 2017). Seja em linha ou em histograma o resultado é o mesmo, visto que os gráficos são gerados a partir de uma fórmula matemática utilizando as médias móveis (THIELE; ADAMI, 2016).

Quando se têm uma média móvel de curto prazo maior que uma de longo prazo é que se obtém a linha do MACD positiva, e com isso tem-se a interpretação de uma tendência de alta; sendo negativa, a tendência passa a ser de baixa. $O$ sinal acaba sendo a média móvel das médias de curto e longo prazo que compõe a linha MACD, sempre por um período menor do que a média móvel de curto prazo. (MATSURA, 2020).

Reproduzimos, na Figura 1, um exemplo do MACD em forma gráfica, sendo a parte superior o histograma e a parte inferior o MACD em sua forma original. O caso refere-se ao ativo BRFS3 (da empresa BRF S.A.) com média móvel longa de 26 dias, curta de 12 dias, e sinal de 9 dias, com o período de 06 de fevereiro de 2018 até 04 de junho de 2018.

Figura 1 - MACD Histograma (BRFS3)

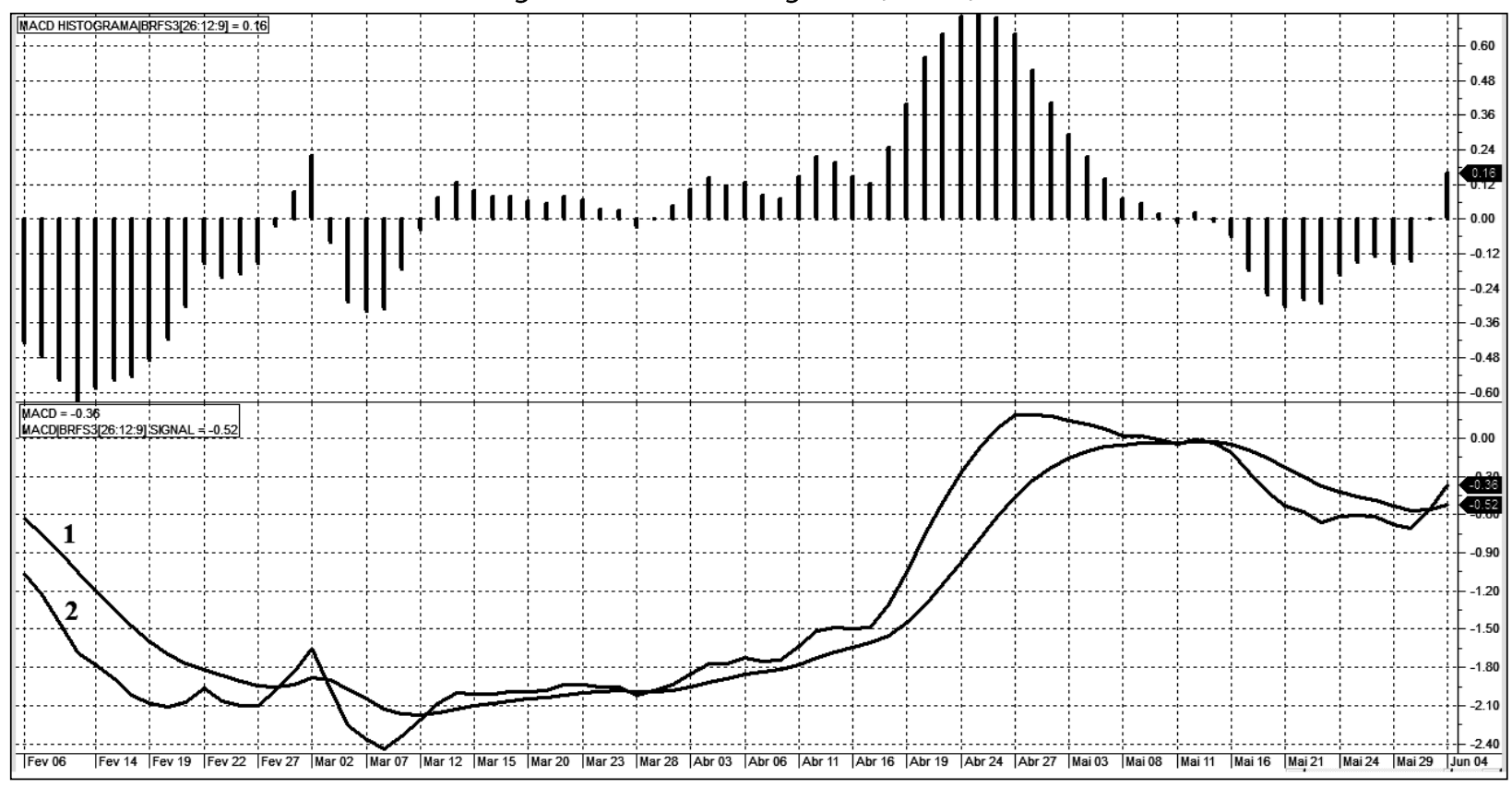

Fonte: Elaborado pelos autores (2021).

A interpretação do histograma é simples, quando as barras estão para baixo é sinal de tendência para baixa, e quando está para cima sinaliza tendência de alta. Já as linhas são representadas pela MACD (linha 2) e o sinal (linha 1), de modo que quando a linha MACD cruza para cima é sinal de compra, e ao cruzar para baixo é de venda.

\subsection{Estudos anteriores sobre o MACD}

Com a finalidade de ampliar ainda mais o embasamento acerca do tema, tem-se a possibilidade de entender como o assunto vem sendo tratado por outros pesquisadores. Para tanto, buscou-se junto aos principais congressos da área estudos comparativos ao aqui realizado. $O$ resultado obtido está apresentado no Quadro 1. 
Quadro 1 - Estudos anteriores

\begin{tabular}{|c|c|c|c|}
\hline AUTOR/ANO & OBJETIVO & METODOLOGIA & RESULTADOS \\
\hline $\begin{array}{l}\text { Vidotto, Migliato e } \\
\text { Zamboni (2009) }\end{array}$ & $\begin{array}{l}\text { Avaliar a eficácia do } \\
\text { MACD em indicações de } \\
\text { tendências de } \\
\text { compra/venda de cinco } \\
\text { empresas. }\end{array}$ & $\begin{array}{l}\text { Pesquisa explicativa } \\
\text { analisando o nível de } \\
\text { acerto do instrumento } \\
\text { MACD em cinco } \\
\text { empresas. }\end{array}$ & $\begin{array}{l}\text { O instrumento foi eficaz } \\
\text { nas análises realizadas. }\end{array}$ \\
\hline Wang et al. (2016) & $\begin{array}{l}\text { Aprimorar o uso de } \\
\text { médias móveis no uso } \\
\text { de petróleo futuro. }\end{array}$ & $\begin{array}{l}\text { Pesquisa explicativa } \\
\text { verificando uma forma } \\
\text { alternativa de uso do } \\
\text { MACD. }\end{array}$ & $\begin{array}{l}\text { A média móvel } \\
\text { dinâmica, como } \\
\text { alternativa à estática, } \\
\text { obteve desempenho } \\
\text { excelente. }\end{array}$ \\
\hline Chaves (2017) & $\begin{array}{l}\text { Analisar MACD, IFR e } \\
\text { Bandas de Bollinger para } \\
\text { tomadas de decisões na } \\
\text { compra e venda de } \\
\text { ações em um mercado } \\
\text { hipoteticamente } \\
\text { eficiente. }\end{array}$ & $\begin{array}{l}\text { Pesquisa explicativa } \\
\text { utilizando ações do } \\
\text { índice Bovespa. }\end{array}$ & $\begin{array}{l}\text { Partindo de um mercado } \\
\text { eficiente, a tomada de } \\
\text { decisão por meio de } \\
\text { indicadores técnicos } \\
\text { demonstrou-se } \\
\text { ineficiente. }\end{array}$ \\
\hline
\end{tabular}

Fonte: Elaborado pelos autores (2021).

Fica claro que, dentre os objetivos dos três estudos encontrados, o objetivo convergente, procurando melhorar e estimar as contribuições de indicadores e ferramentas de gestão dos indicadores para otimizar as decisões de compra e venda no mercado de ações. Quanto a metodologia, foi unânime. Os três estudos utilizam dados empíricos, longitudinais para testar e/ou verificar alternativas do uso de MACD. Como contribuições resultantes dos estudos, foram paralelos, no entanto, com diferenças. Enquanto Vidotto, Migliato e Zamboni (2009) avaliaram apenas o MACD como instrumento, e encontraram eficácia de seu uso. Já Wang et al. (2016), investigaram uma alternativa e viram que, o método dinâmico, assim como o estudo aqui realizará, provocou desempenho excelente na carteira investigada. Por fim, Chaves (2017) usando o ano de 2016, encontrou que a gestão passiva, onde os agentes têm apenas informações sobre o histórico de preços das ações, e seus comportamentos é claro.

\section{MÉTODO}

Para obter uma resposta para a questão problema e alcançar o objetivo deste artigo foram analisados os dados de ações que compõem um determinado índice e, em seguida, comparados com a intensidade da volatilidade em um mesmo período. Tem-se, portanto, que essa é uma pesquisa descritiva, sendo elaborada através de análise documental e análise quantitativa dos dados. Neste viés, pretende-se verificar se há relação entre as oscilações de maior ou menor intensidade de um ativo no mercado mobiliário durante período de 12 meses (julho de 2017 até junho de 2018), com indicações de tendências acertadas pelo MACD; e, havendo essa relação, se há um melhor desempenho no retorno obtido.

Foi examinada uma série histórica das empresas que compõem o índice IBrx 100, separando-as em dois grupos, sendo as $20 \%$ mais voláteis e as $20 \%$ das menos voláteis.

Portanto, a amostra da pesquisa (Quadro 2) foi composta por 40 ações, 20 com maior volatilidade e 20 com menor volatilidade, no mercado de ações brasileiro. A volatilidade foi obtida através do site da B3 utilizando a medida EWMA anualizada, tanto a composição da carteira do IBrX 100 quanto a volatilidade foram obtidas no dia 30 de junho de 2018. 
Quadro 2 - Amostra da pesquisa

\begin{tabular}{|c|c|c|c|c|c|c|c|}
\hline \multicolumn{4}{|c|}{ ATIVOS MAIS VOLÁTEIS (GRUPO 1) } & \multicolumn{4}{|c|}{ ATIVOS MENOS VOLÁTEIS (GRUPO 0) } \\
\hline \# & Código & Nome da Empresa & Volatilidade (\%) & $\#$ & Código & Nome da Empresa & Volatilidade (\%) \\
\hline 1 & ELET3 & ELETROBRAS & 87,53 & 1 & ITUB4 & ITAÚ UNIBANCO & 26,19 \\
\hline 2 & ELPL3 & ELETROPAULO & 73,83 & 2 & KLBN11 & KLABIN & 26,17 \\
\hline 3 & JBSS3 & JBS & 72,57 & 3 & CIEL3 & CIELO & 25,94 \\
\hline 4 & ELET6 & ELETROBRAS & 67,69 & 4 & WEGE3 & WEG & 24,77 \\
\hline 5 & MGLU3 & MAGAZINE LUIZA & 64,83 & 5 & ITSA4 & ITAUSA & 24,06 \\
\hline 6 & GOLL4 & $\mathrm{GOL}$ & 62,46 & 6 & ENBR3 & ENERGIAS BR & 24,05 \\
\hline 7 & LIGT3 & LIGHT & 55,31 & 7 & TAEE11 & TAESA & 23,74 \\
\hline 8 & GOAU4 & GERDAU & 53,3 & 8 & TIET11 & AES TIETE & 23,69 \\
\hline 9 & CSNA3 & SID NACIONAL & 52,36 & 9 & ALUP11 & ALUPAR & 23,42 \\
\hline 10 & WIZS3 & WIZ & 51,78 & 10 & RADL3 & RAIADROGASIL & 23,01 \\
\hline 11 & SUZB3 & SUZANO PAPEL & 51,49 & 11 & HYPE3 & HYPERMARCAS & 22,23 \\
\hline 12 & BTOW3 & B2W DIGITAL & 50,86 & 12 & TIMP3 & TIM PART S/A & 22,22 \\
\hline 13 & CMIG4 & CEMIG & 49,63 & 13 & UGPA3 & ULTRAPAR & 21,74 \\
\hline 14 & AZUL4 & AZUL & 49,37 & 14 & EQTL3 & EQUATORIAL & 21,16 \\
\hline 15 & ESTC3 & ESTACIO & 48,55 & 15 & TRPL4 & TRAN PAULIST & 20,12 \\
\hline 16 & BRSR6 & BANRISUL & 48,28 & 16 & IRBR3 & IRBBRASIL & 18,96 \\
\hline 17 & RAPT4 & RANDON & 47,71 & 17 & VIVT4 & TELEFONICA & 18,36 \\
\hline 18 & USIM5 & USIMINAS & 47,16 & 18 & ABEV3 & AMBEV S/A & 16,86 \\
\hline 19 & VVAR11 & VIAVAREJO & 45,94 & 19 & EGIE3 & ENGIE BRASIL & 16,75 \\
\hline 20 & BRAP4 & BRADESPAR & 45,2 & 20 & CPFE3 & CPFL ENERGIA & 3,68 \\
\hline
\end{tabular}

Fonte: Elaborado pelos autores (2021) com base nos dados primários da pesquisa.

Em seguida foram simuladas as tomadas de decisões em sinais de compra e de venda do ativo, buscando obter a melhor performance possível e checar se um grupo terá melhor performance em relação ao outro. Das empresas que compõem o índice $\mathrm{IBrX} 100$ da B3, o grupo 0 corresponde as empresas menos voláteis e o grupo 1, as empresas mais voláteis.

Na sequência analisaram-se, com base no MACD do período de 01 de julho de 2017 até 29 de junho de 2018, cada empresa, observando pontos de compra e de venda, registrando a data de entrada, o valor da compra, a data da saída e o valor da venda. Nas simulações o MACD foi configurado com os padrões de média móvel longa de 26 dias, curta de 12 dias, e sinal de 9 dias, com período do gráfico diário e os valores de compra e venda foi o do fechamento do dia seguinte ao cruzamento do MACD. Nas simulações foi considerada a diferença entre o valor de compra e venda dos ativos.

A empresa SAPR11 (SANEPAR) não possuía 12 meses de histórico junto à bolsa de valores, motivo pelo qual foi retirada da análise e fora incluída a empresa imediatamente da sequência. Para fins de benchmarking será utilizado o resultado do índice IBOVESPA do mesmo período em que houve a compra e venda, desse modo será possível identificar se o desempenho obtido nas simulações propostas por este trabalho foi melhor do que a do benchmarking utilizado (IBOVESPA).

A análise dos dados se dividiu em análise descritiva, apresentando os resultados de médias simples e na sequência a Análise de Variância (ANOVA) onde foram realizadas as análises de significância. Martins e Domingues (2014) destacam que a ANOVA busca através de testes verificar se uma variável de interesse tem ou não significância sobre a outra, desta forma tornando a comparação estatisticamente comprovada. Foi assumido como significante valores de $p<, 05$. Para os cálculos foram utilizados os softwares Excel e Stata/MP 13. 


\section{ANÁLISE DOS DADOS E DISCUSSÃO DOS RESULTADOS}

Neste tópico serão demonstrados os resultados atingidos com base nas simulações de compra e venda, bem como o desempenho dos grupos de ativos entre si e em relação ao benchmarking (índice Bovespa).

\subsection{Análise descritiva}

Ao todo foram realizadas 278 simulações de compra e venda dos ativos, resultando em 151 no grupo 0 (menos voláteis), e 127 no grupo 1 (mais voláteis). Notou-se que os ativos menos voláteis têm maior sinalização de compras com base no $M A C D$, porém, resta saber se existem 7 pontos em falso, ou seja, sinalização de tendência para alta e não ocorrência no caso concreto.

No grupo 0 a média de dias posicionados em um ativo foi de 19, enquanto no grupo 1 a média foi de 24 dias. Apesar dos ativos do grupo 0 possuírem menor volatilidade, o tempo posicionado nestes ativos através da análise é sensivelmente menor, corroborando a informação do maior número de lançamentos. $O$ grupo 0 apresentou retorno maior que o benchmarking em 31,13\% dos lançamentos, enquanto o grupo 1 foi de $42,52 \%$. Além disto, se dividirmos por ativos, ou seja, das vinte empresas de cada grupo, identificar qual o percentual delas teve retorno maior que o índice Bovespa, tem-se que $40 \%$ das empresas do grupo 0 obtiveram retorno maior, e $75 \%$ no caso das empresas do grupo 1.

Desconsiderando o benchmarking e analisando apenas se o grupo de ativos teve lucro ou prejuízo, constatou-se que o grupo 0 obteve 37,09\% de lucro nas operações, e o grupo 1 apresentou 45,67\%. Usando a mesma ideia do parágrafo anterior, de divisão em grupo de ativos, o grupo 0 teve $40 \%$ das empresas com retorno positivo e o grupo 1 obteve êxito em $85 \%$ dos ativos. Evidentemente o fator de maior interesse é saber o retorno médio de todos os lançamentos separados por grupo, bem como se a performance foi maior ou menor do que o benchmarking. Assim, o grupo 0 obteve uma média de $-0,28 \%$ em todos os lançamentos, enquanto o índice Bovespa teve uma média de $0,63 \%$, ou seja, os lançamentos deste grupo resultaram em prejuízo para o investidor, mesmo tendo o índice um pequeno ganho.

O grupo 1, por sua vez, obteve retorno de 5,46\% ao longo do período, enquanto o índice apresentou retorno de 2,49\%. Dessa forma, destaca-se que o grupo 1 superou em $119,84 \%$ o índice no mesmo período.

\subsection{Análise de variância}

Para aumentar a robustez dos resultados ora apresentados, optou-se por fazer uma análise de variância entre os grupos propostos. A ANOVA permite encontrar diferenças estatisticamente significativas entre grupos de comparação. No caso deste estudo, os resultados apresentam o perfil, expresso na Tabela 3, quando analisadas todas as simulações.

Tabela 3 - Resumo de Lucro/Prejuízo

\begin{tabular}{cccc}
\hline GRUPO & RESULTADO & DESVIO PADRÃO & FREQUÊNCIA \\
\hline 0 &,- 00304636 &, 06012202 & 151 \\
1 &, 05456693 &, 20212662 & 127 \\
\hline Total & $\mathbf{, 0 2 3 2 7 3 3 8}$ & $\mathbf{, 1 4 6 1 7 7 7 9}$ & $\mathbf{2 7 8}$ \\
\hline
\end{tabular}

Fonte: Elaborado pelos autores (2021).

É possível identificar que no grupo 0 as simulações apresentaram resultado negativo de $0,3 \%$ com desvio padrão de $6,01 \%$, enquanto no grupo 1 o resultado foi de $5,45 \%$ com desvio padrão de $20,21 \%$. Estes dados demonstram que de fato o grupo mais volátil apresenta melhor retorno no uso do MACD.

Quando incluído o viés científico (Tabela 4) é importante analisar a variabilidade amostral a fim de identificar se os resultados obtidos são de fato significantes motivos pelo qual se apresenta o quadro de variância. 
Tabela 4 - Análise de variância de Lucro/prejuízo

\begin{tabular}{ccccc}
\hline SS & DF & MS & F & PROB $>$ F \\
\hline 22897137 & 1 &, 22897137 & 11,11 & 0,0010 \\
\hline \multicolumn{4}{c}{ Fonte: Elaborado pelos autores (2021). }
\end{tabular}

Nota-se que o erro amostral é de 0,0010, portanto pode-se considerar os resultados apresentados confiáveis estatisticamente, tendo em vista que a academia considera significantes aqueles de até 0,05 no intervalo de confiança.

Também foram realizadas as análises do ganho sobre o Ibovespa (Tabela 5), salientando que os valores doravante apresentados são pontos percentuais acima do lbovespa e não porcentagem como no caso anterior.

Tabela 5 - Resumo de performance sobre Ibovespa

\begin{tabular}{cccc}
\hline GRUPO & RESULTADO & DESVIO PADRÃO & FREQUÊNCIA \\
\hline 0 &,- 00927152 &, 0476529 & 151 \\
1 &, 0296063 &, 18749138 & 127 \\
\hline Total & $\mathbf{, 0 0 8 4 8 9 2 1}$ & $\mathbf{, 1 3 2 6 5 0 9 1}$ & $\mathbf{2 7 8}$ \\
\hline
\end{tabular}

Fonte: Elaborado pelos autores (2021).

Neste cenário os resultados se mantêm com a mesma lógica, tendo o grupo 0 um resultado de -0,92 pontos percentuais em relação ao Ibovespa, enquanto o grupo 1 apresenta retorno de 2,96 pontos percentuais, ou seja, em um cenário hipotético em que o lbovespa tenha rentabilidade de $1 \%$ o grupo 0 terá 0,08\% e o grupo 1 terá 3,96\%. A análise estatística da performance foi realizada e é ilustrada na Tabela 6.

Tabela 6 - Análise de variância de performance sobre Ibovespa

\begin{tabular}{ccccc}
\hline SS & DF & MS & F & PROB > F \\
\hline 104265281 & 1 &, 104265281 & 6,03 & 0,0147 \\
\hline
\end{tabular}

Fonte: Elaborado pelos autores (2021).

Conforme a Tabela 6 , do ponto de vista de significância estatística, o resultado da variância da performance sobre o lbovespa é de $0.0147(p<, 05)$, sendo considerado estatisticamente significante.

Por fim, nas Tabelas 7 e 8, analisaram-se os dados retirando os extremos (outliers), ou seja, resultados que por algum motivo se tornam atípicos e poderia de alguma forma prejudicar as análises efetuadas. Manter os outliers poderia gerar resultados distorcidos das estimativas, também pode distorcer o valor da média, ou seja, altera o padrão encontrado dos dados e compromete a confiabilidade dos resultados encontrados (FIGUEIREDO FILHO; JÚNIOR, 2009).

Tabela 7 - Resumo de performance sobre Ibovespa

\begin{tabular}{cccc}
\hline GRUPO & RESULTADO & DESVIO PADRÃO & FREQUÊNCIA \\
\hline 0 &,- 00765101 &, 0458315 & 149 \\
1 &, 0108547 &, 09169451 & 117 \\
\hline Total & $\mathbf{, 0 0 0 4 8 8 7 2}$ & $\mathbf{0 7 0 2 7 2 6 9}$ & $\mathbf{2 6 6}$ \\
\hline
\end{tabular}

Fonte: Elaborado pelos autores (2021).

Evidenciou-se que mesmo retirando os extremos, o grupo 0 permanece tendo performance menor do que o Ibovespa em 0,76 pontos percentuais, enquanto o grupo 1 supera o índice em 1,08 pontos percentuais, mantendo o erro amostral abaixo de 0.5 e desta forma fica evidente que o MACD se mostra mais eficiente em empresas mais voláteis. 
Tabela 8 - Análise de variância de performance sobre Ibovespa

\begin{tabular}{ccccc}
\hline SS & DF & MS & F & PROB > F \\
\hline, 022444083 & 1 &, 022444083 & 4,61 & 0,0328 \\
\hline \multicolumn{4}{c}{ Fonte: Elaborado pelos autores (2021). }
\end{tabular}

Os resultados obtidos, são baseados em fatos históricos e com comprovação estatística, forma pela qual a maior parcela do mercado se baseia. No entanto, conforme Taleb (2017) expõe, diversos fatos podem acontecer em um mercado em que os participantes não cogitam a possibilidade de ocorrer. Com isso, se faz relação ao mercado em si, ou seja, até que um evento não ocorra - mesmo que se assume probabilidade (HAIR et al., 2009) - se presume que ele não existe. Por outro lado, mesmo que as amostras coletadas neste trabalho direcionam para um melhor retorno do MACD nos ativos de maior volatilidade, a afirmação não está imune a eventos atípicos.

Como experienciado, o grupo 1 levou vantagem. O grupo mais volátil apresentou rentabilidades maiores no uso do MACD. O resultado vai ao encontro de Vidotto, Migliato e Zamboni (2009) confirmando a eficácia do uso do MACD. Por outro lado, expande o estudo de Chaves (2017) que avaliou compras e vendas, sem divisão por grupos pela volatilidade em 2016, ao evidenciar que em anos subsequentes (2017 e 2018), já que Chaves (2017) se baseou em simulações de compra e venda em 2016, o uso de indicador técnico para o grupo de ativos mais voláteis apresenta melhor desempenho de rentabilidade.

\section{IMPLICAÇÕES PRÁTICAS PARA OS PROFISSIONAIS DE FINANÇAS}

Administradores do capital financeiro de empresas muitas vezes são traders técnicos. Outro grupo destes estão baseados em grandes financeiras, também denominados consultores financeiros que operam valores de propriedade de empresas ou até mesmo por investidores individuais. Ambos baseiam sua análise na premissa de que os padrões nos preços de mercado são supostos se repetir no futuro, e assim, esses padrões podem ser usados para propósitos preditivos. A motivação por trás da análise técnica é ser capaz de identificar mudanças nas tendências em um estágio inicial e manter uma estratégia de investimento para obter retornos positivos (GENCAY, 1998).

Exclusivamente sobre a técnica de média móveis, Gunasekarage e Power (2001) analisam o desempenho de diferentes médias móveis para quatro mercados emergentes de capital do sul da Ásia. Eles encontram evidências claras sugerindo que os retornos de ações nesses mercados são previsíveis. Em relação ao mercado acionário brasileiro, os estudos de Ratner e Leal (1999) e Hatgioannides e Mesomeris (2007) possuem resultados diferentes, mostrando uma falta de consenso.

Cacique da Costa et al. (2015) consideram diferentes regras técnicas de negociação para ações negociadas no mercado acionário brasileiro, enquanto Sobreiro et al. (2016) fazem o mesmo para os BRICS e mercados emergentes. Em ambos os casos, novamente vimos resultados diferentes. Cacique da Costa et al. (2015) constatam que apenas uma pequena parcela dos resultados justifica a utilização das medias móveis. Por outro lado, Sobreiro et al. (2016) concluem que as médias móveis são as estratégias com melhores resultados em países como Brasil, Rússia e Argentina.

Este estudo empregou um dos mais populares indicadores das regras técnicas de negociação, a Convergência e Divergência de Médias Móveis, na análise de dados do índice IBrX 100 da B3. Os fatores econômicos elencados, sobretudo a queda da taxa Selic e a entrada de novos players no mercado financeiro, não apenas são motivadores para as empresas ingressarem na bolsa de valores, mas também refletem o aumento de novos investidores, que buscam novas formas de investimento, em detrimento da queda nas taxas de retorno de aplicações financeiras mais convencionais, especialmente aquelas atreladas a taxa Selic, para potencializar os ativos investidos. De forma geral, o estudo implica na demonstração da estratégia de investimento de utilização da volatilidade como moderador da eficiência da análise gráfica.

O princípio básico da análise técnica é que os padrões relacionados aos preços passados dos instrumentos negociados nos mercados de ativos podem ser usados para prever a direção dos preços futuros. O objetivo é melhorar o retorno de uma carteira de investimentos, entendendo a interação dos indicadores de 
preço para as participações da carteira durante um período identificado. A análise técnica usa uma abordagem sistemática e gráfica para identificar padrões de preços históricos de negociação e movimentos de mercado. O resultado esperado é a possibilidade de formular previsões que podem gerar retornos financeiros anormalmente fortes. (SOUZA et al., 2018)

A partir dos resultados apresentados, mostra-se que é necessária a seleção das ações que compõem o portfólio, utilizando estratégia de investimento e ferramentas de análise como suporte ao critério de decisão. Os resultados obtidos corroboram ainda com possibilidade do desenvolvimento de ferramentas eficazes para gerenciamento de carteira de ações, das pequenas às grandes corporações, a partir do indicador MACD com moderação da volatilidade.

\section{CONCLUSÃO E CONTRIBUIÇÕES DO ESTUDO}

A pesquisa tem como objetivo estimar a relação entre a intensidade da volatilidade de um ativo e a eficiência do instrumento MACD, a fim de colaborar com consultores financeiros que atuam com investimentos de empresas e de investidores individuais, e para pequenos investidores, essencialmente aqueles que estão iniciando no mercado de renda variável.

Nos resultados obtidos torna-se cristalino que no caso do MACD os ativos mais voláteis mostraram-se mais lucrativos do que os menos voláteis, ou seja, apesar de empiricamente os neófitos neste mercado buscarem investir em ativos menos voláteis, o resultado é inversamente proporcional. Este fato confirma a hipótese do objetivo geral de que existe relação entre a intensidade da volatilidade e a eficiência do $M A C D$.

O presente estudo delimitou-se em analisar ativos do mercado de ações brasileiro, sendo empresas listadas no índice IbrX100, separando-as em partes pelo critério de intensidade da volatilidade. No meio acadêmico notou-se pouca abordagem por análises deste tipo, fato que possibilita diversos campos para pesquisas futuras. No decorrer da pesquisa, por escolha dos autores, foram escolhidos dois grupos, dos ativos e dos menos voláteis. Apesar de identificado a máxima no mercado financeiro de que "quanto maior a volatilidade maior o risco", pois a volatilidade é uma medida de risco; não significa que para toda e qualquer técnica de análise é obrigatória a inclusão dos ativos de menor volatilidade são os mais acertados. Porém, o investidor iniciante, por vezes, pode presumir erroneamente que escolher ativos pouco voláteis e utilizar a técnica MACD para análise é a melhor escolha.

A partir dos resultados, descobrimos que existem oportunidades de lucro para usuários da ferramenta MACD. É sabido que, nos últimos anos, com a Selic em queda (KOHLER; PARTYKA; LANA, 2021), os investidores e aspirantes, procuraram potencializar seus ganhos por meio do mercado de ações, portanto é pertinente levar luz a indicadores que ajudam a consolidar o mercado de capitais nacional. Logo, aponta-se que o indicador MACD pode ser utilizado como ferramenta que auxilia os investidores na escolha de ações para potencializar seus ganhos. Além disso, são trazidas evidências de que os resultados deste artigo serão úteis para aplicações reais, no dia a dia de investidores domésticos, traders entre outros. De modo oportuno, os usuários do mercado de ações podem utilizar mais uma ferramenta, seja Índice de Força Relativa, o On Balance Volume, ou o Estocástico entre outros, e assim compará-los instantaneamente.

Por fim, sugere-se para pesquisas futuras com base na volatilidade análises de outros índices, outros ativos financeiros, outros instrumentos de análise técnica. Ainda assim é possível realizar diversas pesquisas com outros fatores além da volatilidade, como nível de governança corporativa, setores de mercado ou em blue-chip, mid-caps ou small-caps. Pode-se também, utilizar indicadores para análise de ações, com um agrupamento por características semelhantes entre empresas, de mesmo setor, por exemplo. Há também a alternativa de considerar o período de aplicação não em dias ou meses, mas em horas dentro de um mesmo dia, assim utilizando o indicador para tomada de decisão no curtíssimo prazo. Também poderiam ser explorados, por meio do uso do MACD, os mercados de futuros, mercado cambial e de renda fixa. 


\section{REFERÊNCIAS}

ADEWUYI, A. Q. Modelling Stock Prices with Exponential Weighted Moving Average (EWMA). Journal of Mathematical Finance, v. 6, p. 99-104, 2016.

AUGUSTO, C.; SILVA, T. O Que Move o Preço da Ação? Uma Abordagem sobre a Influência das Notícias no Mercado Acionário. REUNIR - Revista de Administração, Contabilidade e Sustentabilidade, v. 2, n. 3, p. 113, 2012.

B3 - BRASIL, BOLSA, BALCÃO. Website B3. [2021]. Disponível em: http://www.b3.com.br. Acesso em: 15 jan. 2021.

BETANCOURT BEJARANO, K.; GARCÍA DÍAZ, C. M.; LOZANO RIAÑO, V. Teoría de Markowitz con metodología EWMA para la toma de decisión sobre cómo invertir su dinero. Atlantic Review of Economics, v. 1, n. 1, 2013.

BODIE, Z; KANE, A.; MARCUS, A. J. Fundamentos de investimentos. Porto Alegre: Bookman, 2018.

CACIQUE DA COSTA, T.; NAZÁRIO, R. T.; BERGO, G.; SOBREIRO, V.; KIMURA, H. Trading system based on the use of technical analysis: A computational experiment. Journal of Behavioral and Experimental Finance, v. 6 , p. 42-55, 2015.

CAVALERI, R.; RIBEIRO, E.; RIBEIRO, E. Combinação de Previsões de Volatilidade: Um Estudo. Economia, v. 12, n. 2, 2011

CHAVES, J. P. B. Q. Análise de Indicadores Técnicos para Tomada de Decisão no Mercado de Ações. Monografia - Faculdade de Economia e Administração, Insper Instituto de Ensino e Pesquisa, São Paulo, 2017.

DHAR, V.; STEIN, R. M. FinTech Platforms and Strategy. MIT Sloan School Working Paper, p. 1-19, 2017.

FIGUEIREDO FILHO, D. B.; JÚNIOR, J. A. S. Desvendando os Mistérios do Coeficiente de Correlação de Pearson (r). Revista Política Hoje, v. 18, n. 1, p. 115-146, 2009.

FORTUNA, Eduardo. Mercado Financeiro. São Paulo: Qualitymark, 2019.

GENCAY, R. The predictability of security returns with simple technical trading rules. Journal of Empirical Finance, v. 5, n. 4, p. 347-359, 1998.

GUNASEKARAGE, A.; POWER, D. M. The profitability of moving average trading rules in South Asian stock markets. Emerging Markets Review, v. 2, n. 1, p. 17-33, 2001.

HAIR, J. F. et al. Análise Multivariada de Dados. 6. ed. Porto Alegre: Bookman, 2009.

HATGIOANNIDES, J.; MESOMERIS, S. On the returns generating process and the profitability of trading rules in emerging capital markets. Journal of International Money and Finance, v. 26, p. 948-973, 2007.

KOHLER, J. V.; PARTYKA, R. B.; LANA, J. A selic caiu, e agora? Revista Pretexto, v. 22, n. 1, p. 134-152, 2021.

LO, A. W.; MAMAYSKY, H.; WANG, J. Foundations of technical analysis: Computational algorithms, statistical inference, and empirical implementation. Journal of Finance, v. 55, n. 4, p. 1705-1770, 2000.

MARTIN, K. A.; SBICCA, A. Decisões Financeiras e o Uso de Aplicativos: Um Estudo à Luz da Economia Comportamental. Revista Gestão Organizacional, v. 14, n. 2, p. 207-228, 2021.

MARTINS, G. A.; DOMINGUES, O. Estatística Geral e Aplicada. 5. ed. São Paulo: Atlas, 2014. 
MARTINS, M. V. A.; RODRIGUES, C. A. Uma Estratégia de Investimento Baseada na Divergência do Indicador MACD. Revista de Administração, Contabilidade e Economia da Fundace, v. 9, n. 2, p. 46-59, 2018.

MATSURA, E. K. Comprar Ou Vender? Como investir na Bolsa utilizando Análise Gráfica. 8. ed. São José dos Campos: Benvirá, 2020.

MURCIA, F. Dal-Ri et al. Impacto do nível de disclosure corporativo na volatilidade das ações de companhias abertas no Brasil. Revista de Economia e Administração, v. 10, n. 2, p. 196-218, 2011.

PARTYKA, R.; LANA, J.; GAMA, M. A. Um Olho no Peixe e Outro no Gato: Como as Fintechs Disputam Espaço com os Bancos em Época de Juros Baixos. Administração: Ensino e Pesquisa, v. 21, n. 1, p. 146-180, 2 jan. 2020.

PINHO, F. M.; CARMARGOS, M. A.; FIGUEIREDO, J. M. Uma revisão da literatura sobre modelos de volatilidade em estudos brasileiros. Revista de Administração FACES Journal, v. 16, n. 1, p. 9-28, 2017.

RATNER, M.; LEAL, R.P.C. Tests of technical trading strategies in the emerging equity markets of Latin America and Asia. Journal of Banking and Finance, v. 23, p. 1887-1905, 1999.

SAFFI, P. A. C. Análise técnica: sorte ou realidade? Revista Brasileira de Economia, v. 57, n. 4, p. 953-974, 2003.

SILVA, S. W.; GONÇALVES, J. E.; SOUZA, D. V. R.; PEREIRA, W. F.; FONSECA, L. R. O Sistema Financeiro Nacional Brasileiro: Contexto, estrutura e evolução. Revista da Universidade Vale do Rio Verde, v. 14, n. 1, p. 10151029, 2016.

SOBREIRO, V. A.; CACIQUE DA COSTA, T.; NAZÁRIO, R. T.; LIMA E SILVA, J.; MOREIRA, E. A.; LIMA FILHO, A.; KIMURA, H.; ZAMBRANO, J.C. The profitability of moving average trading rules in BRICS and emerging stock markets. North American Journal of Economics and Finance, v. 38, p. 86-101, 2016.

SOUZA, M. J. S. S.; RAMOS, D. G. F.; PENA, M. G.; SOBREIRO, V. A.; KIMURA, H. Examination of the profitability of technical analysis based on moving average strategies in BRICS. Financial Innovation, v. 4, n. 3, p. 1-18, 2018.

TALEB, N. N. A lógica do Cisne Negro: o impacto do altamente improvável. 12. ed. Rio de Janeiro: BestBusiness, 2017.

THIELE, C. C.; ADAMI, A. G. Previsão de séries temporais financeiras: modelo baseado em redes neurais artificiais. Revista Brasileira de Computação Aplicada, v. 8, n. 2, p. 113, 2016.

VAIDYA, R. Moving Average Convergence-Divergence (MACD) Trading Rule: An Application in Nepalese Stock Market "NEPSE”. Quantitative Economics and Management Studies, v. 1, n. 6, p. 366-374, 2020.

VIDOTTO, R. S.; MIGLIATO, A. L. T.; ZAMBON, A. C. O Moving Average Convergence-Divergence como Ferramenta para a Decisão de Investimentos no Mercado de Ações. Revista de Administração Contemporânea, v. 13, n. 2, p. 291-309, 2009.

WANG, L.; AN, H.; LIU, X.; HUANG, X. Selecting dynamic moving average trading rules in the crude oil futures market using a genetic approach. Applied Energy, v. 162, p. 1608-1618, 2016.

WONGLIMPIYARAT, J. FinTech banking industry: a systemic approach. Foresight, v. 19, n. 6, p. 590-603, 2017. 